\title{
The individuality of shape asymmetries of the human cerebral cortex
}

Yu-Chi Chena, ${ }^{a}{ }^{\star}$, Aurina Arnatkeviciute ${ }^{a}$, Eugene McTavisha,b, James Pang ${ }^{a, b}$, Chao Suo ${ }^{a, b}$, Alex Fornito ${ }^{a, b, 1}$, and Kevin M. Aquino ${ }^{a, b, c, d, 1}$, for the Alzheimer's Disease Neuroimaging Initiative ${ }^{2}$

aTurner Institute for Brain and Mental Health, School of Psychological Sciences, Monash University, Victoria, Australia 3800

bMonash Biomedical Imaging, Monash University, Victoria, Australia 3800

'School of Physics, University of Sydney, New South Wales, Australia 2006

${ }^{\mathrm{d} C e n t e r}$ of Excellence for Integrative Brain Function, University of Sydney, Australia 2006

${ }^{1}$ A.F. and K.M.A. contributed equally to this work.

${ }^{2}$ Data used in preparation of this article were obtained from the Alzheimer's Disease Neuroimaging Initiative (ADNI) database (adni.loni.usc.edu). As such, the investigators within the ADNI contributed to the design and implementation of ADNI and/or provided data but did not participate in analysis or writing of this report. A complete listing of ADNI investigators can be found at: http://adni.loni.usc.edu/wp-content/uploads/how_to_apply/ADNI_Acknowledgement_List.pdf

*Yu-Chi Chen

Email: yu-chi.chen@monash.edu

Keywords: brain asymmetry, scale analysis, cortical shape, subject identifiability, heritability

\begin{abstract}
Asymmetries of the cerebral cortex are found across diverse phyla and occur at multiple spatial scales, from the global morphology of the two hemispheres to fine-grained sulcal and gyral anatomy. Cortical asymmetries are particularly pronounced in humans and have important implications for brain function and disease. However, most studies conflate asymmetries in regional size and shape and only consider effects at fairly fine-grained resolutions, such as image voxels or a priori regions-of-interest. As such, whether asymmetries are preferentially expressed at specific spatial resolutions over others remains unclear. Here, we apply a spectral shape analysis to magnetic resonance imaging (MRI) data from three independent samples to derive a multiscale description of asymmetries in cortical shape that is distinct from regional size-related descriptors such as volume or thickness. We show that our proposed shape asymmetry signature (SAS) is a highly individualized feature, akin to a cortical fingerprint, that more accurately identifies individuals than size-based descriptors or measures of inter-regional functional coupling of brain activity. Notably, individual identifiability is optimal at coarse spatial scales ( $\sim 37 \mathrm{~mm}$ wavelength), and shape asymmetries at specific scales show differences between males and females but are unrelated to the handedness. We further demonstrate that while unihemispheric shape descriptors show significant heritability at coarse scales, the SAS are determined primarily by unique environmental effects across all scales considered. Our findings thus identify coarse-scale scale asymmetries of cortical shape as being highly unique to individuals, sexually dimorphic, and largely driven by stochastic environmental influences.
\end{abstract}




\section{Introduction}

Asymmetries in brain structure and function are found throughout the animal kingdom (1-4) and can be discerned at multiple spatial scales, ranging from differences in the size and shape of the cerebral hemispheres through measures of regional morphometry and connectivity to cellular and molecular organization $(2,4,5)$. At the coarsest scale, the most salient feature of anatomical asymmetry in the human brain is cerebral torque, in which the right hemisphere appears to be warped in the rostral direction relative to the left hemisphere (6-8). More fine-grained asymmetries of specific sulci/gyri $(9)$ and brain regions $(10,11)$ have also been investigated. For example, the superior temporal sulcus, which is adjacent to the Wernicke's area, shows a leftward asymmetry in length (12).

Asymmetries in brain organization are often considered at an average level across a population of individuals $(7,10,13-15)$. Also called directional asymmetries $(12,16)$, they have been studied extensively and are thought to have important implications for both functional lateralization and abnormal brain function in a wide range of psychiatric and neurological diseases $(5,11,15,17-25)$. However, the specific regions showing these asymmetries, their directions, and their magnitudes are often inconsistent $(10,11,13)$. The correlates of these asymmetries are also unclear $(4,11,13,26,27)$. For example, two fundamental characteristics often examined in relation to cerebral asymmetry are sex and handedness. Some studies have found that the surface area (10), shape (27-29), volume (30), and torque (8) of cortical structures in males are more asymmetric than in females, whereas other studies have found no sex differences $(31,32)$. Similarly, some studies have found associations between cerebral asymmetry and handedness $(8,14,33)$, with others reporting no such effect $(10,11,28,32,34-36)$.

Some of these inconsistencies may arise from the disparate methodologies and the heterogeneous nature of the brain asymmetries across the population $(7,10,12-15)$. There is often considerable individual variability around population means, with many people often showing little or even reversed asymmetries relative to the group average $(3,12,16,37)$. The distinction between population-level (directional asymmetries) and individual-level asymmetries is essential, as they are thought to arise from distinct mechanisms $(16,38)$. Directional asymmetries are hypothesized to have a genetic basis $(8,10,16,37-42)$, whereas the degree to which a given individual departs from the population mean, referred to as fluctuating asymmetries, may reflect environmental influences, developmental plasticity, or individual-specific genetic perturbations (16, 37-41). Notably, fluctuating asymmetry is more pronounced in humans than other primates, at both regional and global hemispheric levels $(12,37)$. The variability is most evident in regions of heteromodal association cortex, leading some to conclude that high levels of fluctuating asymmetry may have emerged in line with the evolution of human-specific cognition (12). However, traditional analysis methods, which rely on standard image processing techniques such as image registration and spatial smoothing, minimize individual variation and thus have limited sensitivity for studying fluctuating asymmetries $(23,43)$. Moreover, most past works have focused on morphological properties related to the size of specific brain regions, such as estimates of grey matter volume, cortical thickness, or surface area, often measured at fine-grained resolutions, such as individual voxels or the vertices of cortical surface mesh models (17, 26, 31, 34, 36, 44-46). Many of the most obvious features of cerebral asymmetry arise from variations in brain shape, which are not captured by size-related descriptors $(28,47)$. Indeed, it is possible for two objects to have identical volume but have very different shapes $(47,48)$. In addition, shape variations can occur at different spatial resolution scales, from the presence and configuration of specific sulci at fine scales to more global patterns such as cerebral petalia at coarser scales. Conventional analyses only consider the finest 
resolvable scale (i.e., point-wise differences) and have limited sensitivity for identifying important morphological variations that occur over large swathes of cortical tissue.

A comprehensive, multiscale description of cortical shape, from the finest to coarsest scales, can be derived through a spectral analysis of cortical geometry based on solutions to the Helmholtz equation $(28,47,49)$, which is fundamental in many branches of physics, engineering, chemistry, and biology (51). The equation can be solved by formulating it as an eigenfunctioneigenvalue problem of the Laplace-Beltrami operator (LBO) (see Materials and Methods). Importantly, the characteristics of the eigenfunctions and eigenvalues depend on the cortical shape for which the equation is solved $(49,50)$, and thus, the spectral analysis provides a comprehensive description of the intrinsic geometry of a given object, akin to a "Shape-DNA" (49). The application of such Shape-DNA analysis to human magnetic resonance imaging (MRI) data has shown that shape properties of cortical and subcortical structures have superior sensitivity compared to traditional, size-based measures for identifying individual subjects (28), for classifying and predicting the progress of psychiatric and neurological diseases $(23,51)$, and for detecting genetic influences on brain structure $(48,52)$. However, a detailed characterization of fluctuating asymmetries in cerebral shape is lacking.

Here, we introduce methods for constructing an individual-specific measure of fluctuating asymmetries, called the shape asymmetry signature (SAS), that characterizes pure shape asymmetries, independent of variations in size, of the whole cortical surface across a spectrum of spatial scales. We apply this methodology to three independent longitudinal datasets to show that the SAS is a highly personalized and robust feature that can identify individuals, like a cortical asymmetry fingerprint, with greater accuracy than shape descriptors of individual hemispheres, asymmetries in traditional size-based descriptors, or patterns of inter-regional functional connectivity (so-called connectome fingerprinting (53)), thus indicating that cerebral asymmetries in shape are highly unique to individual people. Further, we use the multiscale characterization offered by spectral shape analysis to show that the spatial scales that are optimal for identifying individuals are expressed over coarse spatial wavelengths and not fine-grained variations in sulcal and gyral anatomy. We then show that the SAS does not covary with handedness but does differ between males and females, with some coarse scales showing a stronger rightward shape asymmetry in females than males. Finally, we test the hypothesis that fluctuating asymmetries are largely driven by environmental influences using classical heritability modeling of twin data. We show that while shape descriptors of individual hemispheres at coarse scales are under significant genetic influence, shape asymmetries are primarily influenced by non-shared environmental factors. Together, our results indicate that fluctuating asymmetries in cortical shape are a highly unique and informative property of individual brains that are primarily sculpted by non-genetic mechanisms.

\section{Results}

\section{Cortical shape asymmetries are individually unique}

To understand how cortical shape asymmetries vary across individuals, we examined the degree to which different cortical shape descriptors can be used to identify individual brains from a large sample of T1-weighted magnetic resonance images (MRIs). We analyzed healthy subjects from three open-source datasets - the latest Open Access Series of Imaging Studies (OASIS-3 (54)), the Human Connectome Project (HCP (55)), and the Alzheimer's Disease Neuroimaging Initiative (ADNI; https://ida.loni.usc.edu/) - in which individuals had at least two anatomical MRI scans acquired at different time points (separated by at least one month; see SI Materials and Methods). For each dataset, we asked whether the shape descriptors for an individual estimated from the first scan could accurately identify the same participant's second scan. Within each dataset, the shape descriptor was calculated from the cortical surfaces at the white and grey matter boundary 
estimated either from FreeSurfer (56) (OASIS-3 and ADNI) or FreeSurfer-HCP (HCP), which is a FreeSurfer (56) pipeline with some HCP-specific enhancements (57). Shape-DNA $(47,49)$ analysis was employed to obtain multidimensional shape descriptors for each hemisphere that quantify the shape of each individual's cortex, as defined by the spectrum of eigenvalues of the LBO (Fig. 1 A$B$; see Materials and Methods). Each eigenvalue is associated with a corresponding eigenfunction, which describes shape variations at a particular spatial wavelength, ordered from coarse to finegrained scales (Fig. 1B). These eigenfunctions are orthogonal by construction and thus represent a basis set for cortical shape variations much like the sinusoidal basis used in Fourier decomposition of signals, with the corresponding eigenvalue being analogous to the wave frequency at each spatial scale. Critically, we normalized the surface area (47) of the meshes prior to Shape-DNA analysis to ensure that the resulting eigenvalue spectra were independent of individual differences in brain size (see Materials and Methods).

To investigate the uniqueness of these shape descriptors to individual brains, we performed an identifiability analysis $(58,59)$, where identifiability was quantified as the degree to which the surface eigenspectrum of an individual at scan time 1 was more similar to the same person's eigenspectrum at time 2, relative to other people's time 2 spectra (Fig. 1C; Materials and Methods). To determine whether identifiability is maximized at any specific scales, we repeated the analysis multiple times, initially by taking only the first two eigenvalues, which describe shape variations at the coarsest scale, and then incrementally adding eigenvalues representing more finegrained features to a maximum of 1000 . Plotting the identifiability score as a function of the number of eigenvalues allows us to identify characteristic spatial scales at which the identifiability score is maximized (Fig. 1D). In other words, it allows us to identify the scales at which individual-specific shape features are most pronounced. We repeated this procedure using the eigenvalue spectra for the left and right hemispheres alone, the combination of both (which describes the shape of both hemispheres), and for the SAS, which quantifies shape asymmetries as the difference between the left and right hemisphere eigenspectra (Fig. $2 A-C$ ). Finally, we utilized the spatial scales with the maximum identifiability (Fig. $1 D$ ) to examine the relationships between the SAS and sex, handedness (Fig. 1E) and heritability (Fig. 1F).

Fig. $2 A-C$ shows the identifiability scores obtained for the different shape descriptors. In all three datasets, across a broad range of spatial scales, identifiability was highest for the SAS, followed by the combination of left and right hemisphere eigenvalues, and then each hemisphere alone. This result indicates that individual variability in the asymmetry of cortical shape is greater than the variability of shape across the whole cortex or within each hemisphere alone. Fig. $2 \mathrm{~A}-\mathrm{C}$ also shows identifiability scores obtained when trying to identify an individual's left hemisphere using right hemisphere shape descriptors obtained at the same time point. These scores are very low, indicating that shape variations between the two hemispheres are largely independent of each other and lack a consistent pattern amongst subjects. In other words, for any given person, the shape of one hemisphere offers little individually unique information about the shape of the other hemisphere.

\section{Individually unique variations of cortical shape asymmetry are maximal at coarse spatial scales}

We next investigated the scale-specificity of SAS identifiability. Fig. 2 A-C shows that SAS identifiability sharply increases to a peak as we use more eigenvalues to characterize the surface shape at finer scales (i.e., as we add more shape information from finer spatial scales), before gradually falling again. This peak identifies a characteristic spatial scale in which individual differences in shape asymmetries are maximally unique (see also Fig. S1).

Peak SAS identifiability was observed using the first 126 and 122 eigenvalues for the OASIS-3 (Fig. 2A) and ADNI (Fig. 2B) data, respectively. At these scales, the subject identifiability 
scores were $4.94(P=0)$ for OASIS-3 and $5.10(P=0)$ for ADNI. For the HCP data, peak SAS identifiability was observed when using the first 268 eigenvalues (identifiability score $=6.74 ; P=0$; Fig. 2C), but the identification curve flattened after the first 137 eigenvalues (identifiability score $=$ 6.56), which is closely aligned with the OASIS-3 and ADNI datasets.

In the case of a perfect sphere, the shape spectral analysis yields subsets of degenerate eigenvalues with equal magnitude (60), within which the corresponding eigenfunctions represent orthogonal rotations of the same spatial pattern at a given scale. For example, eigenfunctions 2-4 of a sphere represent coarse-scale gradients in the anterior-posterior, inferior-superior, and leftright axes. As the cortex is topologically equivalent to a sphere, the spherical eigen-groups offer a natural way to identify characteristic spatial scales, to more succinctly summarize cortical shape variations (60), and to smooth out eigenvalue-specific fluctuations at a given scale (see Materials and Methods). We averaged the identifiability scores for each harmonic group and plotted these as a function of the group index in Fig. $2 D-F$. The group mean identifiability score peaks at the $11^{\text {th }}$ eigenvalue group for the OASIS-3 (mean identification score $=4.93$ ) and ADNI (mean identifiability score $=5.06$ ) datasets, which is comprised of the first 144 eigenvalues. Identifiability also reaches a near-plateau at the $11^{\text {th }}$ group for the HCP data (mean identifiability score $=6.47$ ), with an additional marginal increase observed at the $16^{\text {th }}$ group (mean identifiability score $=6.69$ ). Thus, the first 144 eigenvalues represent a stable and robust characteristic scale at which individual uniqueness in cortical shape asymmetry is strongest. The $11^{\text {th }}$ group corresponds to a wavelength of approximately $37 \mathrm{~mm}$ in the case of the population-based template (fsaverage in FreeSurfer; Table S1 shows the corresponding wavelengths of the first 13 eigen-groups; Fig. $2 G$ shows the spatial scales corresponding to the cumulative eigen-groups). A reconstruction of the cortical surface using the first 144 eigenfunctions is shown in Fig. $2 \mathrm{H}$. The reconstruction captures shape variations at a coarse scale, representing major primary and some secondary sulci but with minimal additional details. This same characteristic scale was obtained after repeating the identifiability analysis over the longest inter-scan intervals in the ADNI and OASIS-3 datasets (Fig. S2), indicating that our results are robust over time windows ranging from one month to more than six years.

\section{Cortical shape asymmetries are more identifiable than classical functional and morphological measures}

We next compared the identifiability of the SAS to scores obtained using asymmetries in classical morphological descriptors such as regional surface area, cortical thickness and grey matter volume, and measures of inter-regional functional connectivity (Fig. 3), which have previously been shown to yield high identifiability $(53,58)$. Identifiability scores obtained with the SAS were much higher than those obtained by regional asymmetries in size-related morphological measures with the HCPMMP1 atlas (61) (Fig. $3 A-B$ ). We also found that SAS identifiability was higher when using our surface area normalization procedure compared to the SAS computed without normalization (Fig. S3; see SI Materials and Methods). Since the normalization isolates the pure effects of shape independent of brain size, the results converge to indicate that individual variability in brain anatomy is higher when considering asymmetries in cortical shape compared to more traditional size-based morphological descriptors.

Fig. $3 C-F$ compares the identifiability scores obtained from SAS to those obtained using inter-regional functional connectivity (see SI Materials and Methods), within the HCP test-retest data. Functional connectivity was quantified for the entire cortex using four different regional parcellations defined at different spatial scales (Schafer 100, Schaefer 300, Schaefer 900 (62), and HCP-MMP1 (61) atlas). The SAS outperformed all functional identifiability scores, indicating that cortical shape shows greater specificity to an individual than patterns of functional connectivity. 


\section{Cortical shape asymmetries are related to sex but not handedness}

Sex and handedness are two characteristics that have frequently been examined with brain asymmetry $(2,7,10,11,28,30,32,34,63)$. We used a general linear model (GLM) with permutation testing to account for familial structure of the HCP data $(64,65)$ to evaluate the association between these two characteristics and the SAS defined at each eigenvalue ranging between the $2^{\text {nd }}$ and $144^{\text {th }}$. After FDR correction, males and females showed significant differences in asymmetry scores for the $2^{\text {nd }}\left(P_{F D R}=0.037\right), 6^{\text {th }}\left(P_{F D R}=0.037\right), 8^{\text {th }}\left(P_{F D R}=0.039\right), 52^{\text {nd }}\left(P_{F D R}=\right.$ $0.030)$, and $84^{\text {th }}\left(P_{F D R}=0.037\right)$ eigenvalues (Fig. $\left.4 A\right)$, where female brains showed more rightward asymmetric than males in these eigenvalues. These five eigenvalues come from four different eigen-groups, and the corresponding spatial scales of these eigenvalues are shown in Fig. $4 B$. These eigenvalues relate to shape variations over coarse scales. For instance, for the $2^{\text {nd }}$ eigenvalue ( $L=1$; see Materials and Methods for the definition of $L$ ), the wavelength is of order $300 \mathrm{~mm}$, which is about half the circumference of the brain; for the most-fine grained eigenvalue, $\lambda^{84}(L=9)$, the wavelength is about $44 \mathrm{~mm}$. We note however that the sex differences are small, with considerable overlap between male and female distributions (Fig. 4A). No such effects of handedness on the SAS surpassed the FDR-corrected threshold. We also found that the overall asymmetry level (i.e., the sum of the SAS) was not correlated with either handedness or sex.

\section{Cortical shape asymmetries are primarily driven by unique environmental influences}

To characterize genetic and environmental effects on cortical shape and its asymmetry, we calculated the heritability of each eigenvalue within the left and right hemispheres, as well as for the SAS. We used data from 138 monozygotic (MZ) twin pairs, 79 dizygotic (DZ) twin pairs, and 160 of their non-twin siblings drawn from the HCP dataset (55) (see SI Materials and Methods for details). Uni-hemispheric shape descriptors demonstrated strong heritability at very coarse spatial scales and moderate heritability at slightly finer scales. For instance, the heritability of the $1^{\text {st }}$ eigengroup ( $2^{\text {nd }}$ to $4^{\text {th }}$ eigenvalues) of both hemispheres ranged between $0.52<h^{2}<0.69$ (all $P<0.05$ after FDR correction; Fig. $5 A-B$ ). These eigenvalues are related to shape variations on the coarsest scale that does not include any sulcal or gyral features (the corresponding wavelength is approximately $170 \mathrm{~mm}$ ). Beyond the $2^{\text {nd }}$ eigen-group, heritability estimates dropped to below 0.5 $\left(P<0.05\right.$ after FDR correction for most eigenvalues), and beyond the $4^{\text {th }}$ eigen-group they dropped below 0.3. Most eigenvalues with statistically significant heritability estimates were confined to the first six eigen-groups, which correspond to scales larger than $60 \mathrm{~mm}$ (Fig. 5 A-B insets). These results indicate that genetic influences on the shape of each cortical hemisphere are expressed over very coarse scales at which only primary cortical folds such as the Sylvian and central sulci are apparent. Estimates of common environmental influence on both hemispheres were uniformly low across the first 144 eigenvalues (range 0-0.20).

In contrast to unihemispheric shape variations, all the heritability estimates of the SAS were low (<0.28; Fig. $5 C)$, with only four eigenvalues $(2,3,16$, and 28$)$ showing statistically significant heritability after FDR correction $\left(P_{F D R}=0.004\right.$ to 0.022$)$ and no estimates exceeding 0.30 . Thus, at any given scale, genes account for less than one-third of the phenotypic variance in the SAS. These four eigenvalues are confined to the first five eigen-groups, with corresponding wavelengths greater than or equal to $75 \mathrm{~mm}$ (Fig. $5 \mathrm{C}$ inset). Estimates of common environmental influences were uniformly low (range 0-0.14), whereas non-shared environmental influences on the SAS were consistently high across the full range of eigenvalues considered, ranging between 0.72 to 1.00 (Fig. 5D). In line with the high non-shared environmental influences and low heritability of the SAS, we were unable to identify one subject's SAS from his or her MZ twin sibling's SAS (identifiability score $=0.24, P=0.20$ ).

Notably, heritability estimates for non-surface area normalized eigenvalues of individual hemispheres, which capture variations in both shape and size, were uniformly high across all 
scales, and the scale-specific effects were eliminated (Fig. S4), indicating that variations in cortical size are under greater genetic influence than cortical shape. The results underscore the importance of controlling for size-related variations in shape analyses.

\section{Discussion}

Asymmetries in brain anatomy are widely viewed as a critical characteristic for understanding brain function. Here, we employed a multiscale approach to examine fluctuating shape asymmetries of the human cerebral cortex. We found fluctuating asymmetries were highly personalized and robust, with shape asymmetries at coarse spatial scales being the most discriminative among individuals and also showing differences between males and females. Heritability estimates of shape descriptors in individual hemispheres were high at very coarse scales but declined to moderate values at finer scales. By contrast, the heritability of fluctuating asymmetries in shape was low at all scales, with such asymmetries being predominantly influenced by individual-specific environmental factors.

\section{Identifiability of cortical shape asymmetry is maximal at coarse scales}

Cortical asymmetries have traditionally been investigated at fine-scale, voxel or vertex-level resolutions $(17,26,31,34,36,44-46)$. These approaches may ignore meaningful effects at coarser spatial scales. Our SAS quantifies these underlying variations across the whole brain and along a spectrum of spatial scales. Our approach is akin to studying seismic waves of earthquakes with different wave frequencies at the global tectonic scale, instead of focusing on a particular city. The ability to assess shape along a spectrum of spatial scales is important, since brain asymmetry is a multidimensional and multivariate phenotype $(3,13,45)$.

Few studies have assessed shape at coarse scales. Neubauer et al. (37) found that fluctuating asymmetry in endocranial shape is reliable across two time points. The endocranial shape is the imprint of the cortical surface shape but contains only very coarse shape information (37). Moreover, levels of brain torque (both horizontal and vertical) are robust across time (63). Wachinger et al. (28) used shape descriptors at coarse scales derived from the eigenvalues of the LBO for all brain structures to achieve accurate subject identification.

It is perhaps surprising that individual differences in cortical shape are most strongly expressed at coarse scales, given the known variability of fine-grained anatomical features such as the presence and trajectories of secondary and tertiary sulci. It is possible that these fine-grained features are not captured reliably, meaning that they contribute noise to identifiability analyses. Traditional analysis methods use smoothing to address the issue of image noise (46), but smoothing can also suppress the actual variations at fine scales. Our multiscale approach affords a more comprehensive characterization of shape variations and their uniqueness. An important avenue of future work will involve investigating the functional consequences of these pronounced individual differences.

\section{Cortical shape, rather than shape asymmetry, is heritable}

Genetic effects on cortical thickness and surface area are generally bilateral $(10,66-68)$, resulting in few lateralized effects $(10,69)$. Accordingly, it has been postulated that fluctuating asymmetries may be largely determined by environmental factors $(16,38-41)$. In line with this hypothesis, we found that fluctuating asymmetries in cortical shape were associated with low heritability and were driven mainly by unique environmental effects. These environmental effects are captured by the $E$ parameter of the ACTE heritability model that we used in our analysis; this parameter also includes the contributions of measurement error. However, our effects cannot be explained by the SAS being a noisier descriptor of morphology as it showed the highest identifiability (Fig. $2 A-C$ ). That is, a noisy measure will not be able to discriminate between individuals. Thus, taking the findings 
of our identifiability and heritability analyses together, we can conclude that individual differences in SAS scores are primarily driven by unique environmental influences rather than measurement error. Previous studies have found some evidence of environmental influences on brain asymmetry $(2,4,5,70)$. Early in the intrauterine environment, fetal posture and light may influence brain asymmetry $(2,4,5)$; during postnatal maturation, language learning has been linked to specific asymmetry features. For example, bilinguals have stronger rightward asymmetry of cortical thickness of the anterior cingulate than monolinguals (70). However, the mechanisms of how environmental effects shape brain asymmetry are largely unknown, and epigenetics may also play a role $(2,4)$.

In contrast to shape asymmetries, the shape of individual hemispheres showed greater heritability at coarse scales, consistent with results from previous studies on other morphological measurements $(10,42,45)$. The scales at which genetic effects on unihemispheric shape were observed captured variations in primary sulci, consistent with evidence that the primary folds, which develop early in life, are less variable across individuals and under greater genetic control than other folds (i.e., secondary and tertiary folds) $(45,71,72)$. Previous studies have found that genetic influences on the cerebral thickness, geodesic depth, and surface curvature generally occur along the posterior-anterior and inferior-superior axes $(45,73)$. These two axes correspond to the second and third eigenvalues of the LBO, which also showed strong heritability in the shapes of both hemispheres in our results. In addition to these two axes, we found strong heritability at very coarse scales in other directions that have not been described in previous studies. Our approach thus identifies dominant spatial scales and gradients of heritability in shape.

\section{Shape asymmetries, sex, and handedness}

Using our multiscale approach, we did not find a relationship between shape asymmetry and handedness, consistent with numerous studies showing that handedness is unrelated to anatomical brain asymmetry in cortical thickness, volume, surface area, shape, and voxel-based morphometric (VBM) analysis (10, 11, 27, 28, 32, 34, 36).

Numerous studies, focusing primarily on size-related descriptions such as grey matter volume and cortical thickness, have found that female brains are more symmetric than male brains $(8,10,27-30)$. Our analysis reveals that, although the overall level of shape asymmetry did not differ between male and female brains, female brains displayed a greater rightward shape asymmetry than male brains at certain coarse spatial scales, such as along the anterior-posterior axis. The mechanisms giving rise to these scale-specific sex differences require further investigation.

\section{Conclusions}

We developed a multiscale approach for characterizing individualized asymmetries of the human cerebral cortex. We found that fluctuating asymmetries in cortical shape are robust and personalized neuroanatomical phenotypes, especially at coarse spatial scales. Some of these coarse scales are more strongly rightward asymmetric in females compared to males. Finally, fluctuating asymmetries in shape are driven mainly by environmental influences rather than by genetics, unlike the shape of individual hemispheres that shows strong heritability at coarse scales.

\section{Materials and Methods}




\section{Neuroimaging data}

We used healthy subject data from three open-source neuroimaging datasets (see SI Materials and Methods for details): the latest Open Access Series of Imaging Studies (OASIS-3 (54)), the Human Connectome Project (HCP (55)), and the Alzheimer's Disease Neuroimaging Initiative (ADNI; https://ida.Ioni.usc.edu/) to develop and test our new asymmetry shape measure - the SAS (see below for details). To test for relationships of sex, handedness, and heritability, we restricted our analysis to the HCP dataset, which provides twin and non-twin sibling information and handedness measurement as a continuous variable, as the sample sizes of the left-handers in the other two datasets are too small ( $n=15$ in the ADNI data; $n=18$ in the OASIS-3 data).

\section{Spectral shape analysis}

We utilized the eigenvalues of the LBO applied to cortical surface mesh models generated with FreeSurfer (56). The eigendecomposition of each individual's cortical surface was estimated using the Shape-DNA software $(28,47,49)$, which provides algorithms that extract and optimize the eigenvalues and eigenfunctions from the LBO based on the intrinsic shape of an object $(47,49)$. The Shape-DNA software $(47,49)$ uses the cubic finite element method to solve the Helmholtz equation (Eq. 1), also known as the Laplacian eigenvalue problem:

$\Delta f=-\lambda f$

where $\Delta$ is the LBO; $f$ is the eigenfunction with corresponding eigenvalue $\lambda$. The eigenvalues of the Helmholtz equation are a sequence ranging from zero to infinity, i.e., $0 \leq \lambda^{1} \leq \lambda^{2} \leq \ldots<\infty$, and changes in shape result in changes in the eigenvalue spectrum (49).

Spectral shape analysis via LBO is a departure from traditional morphological analyses that focus on either specific locations (i.e., regions defined by a cortical atlas) or global differences (such as total hemispheric volume). Spectral shape analysis focuses instead on differences in the spatial scales of variation. The decomposed spatial scales can be linearly combined to reconstruct the surface via the eigenfunctions and their corresponding coefficients (the contribution of each set of eigenfunctions to the original surface; see Fig. $2 G$ for examples of reconstructed surfaces).

Importantly, Shape-DNA achieves better results for retrieving object shapes than numerous cutting-edge shape-retrieval methods (74). Shape-DNA compresses the cortical-surface geometry from around $5 \mathrm{mb}$ into only less than $3 \mathrm{~kb}$, making it computationally efficient for further analysis (28). The code for calculating Shape-DNA is written in Python and is freely available (http://reuter.mit.edu/software/shapedna/). We applied the Shape-DNA code to the data and analyzed the resulting eigenvalues using MATLAB. The heritability was estimated using OpenMx software in $\mathrm{R}(75,76)$. Code to reproduce the analyses presented in this study is freely available at https://github.com/cyctbdbw/Shape-Asymmetry-Signature.

\section{Eigenvalue normalization}

To account for differences in brain sizes among participants, the eigenvalue spectra from ShapeDNA should be normalized (47). Previous studies $(23,28,51,52)$ have applied volume normalization to normalize the eigenvalue spectrum to unit volume via the following equation (23, 28):

$\lambda^{\prime}=v^{2 / D} \lambda$

where $v$ is the Riemannian volume of the manifold, $\lambda$ is the original eigenspectrum $\left(\lambda=\left[\lambda^{1}, \lambda^{2}, \ldots\right]\right)$ and $\lambda^{\prime}$ is the volume normalized eigenvalue spectrum. Although this approach has been used in the literature, it is still unable to isolate shape properties as it does not control the effect of different 
surface areas among objects (see SI Materials and Methods for details). Hence, an appropriate surface area-based normalization is essential to isolate the effects of shape that are distinct from size, particularly given the evidence that the right hemisphere tends to have a greater cortical surface area than the left hemisphere (10). Without surface area normalization, differences between the hemispheres may be primarily driven by differences in the surface area of the two hemispheres (SI Materials and Methods; Fig. S7).

To perform surface area normalization, we obtained the unit surface area by dividing the vertex coordinates on each axis by the square root of the total surface area (Eq. 3).

$\mathrm{Vx}^{\prime}=\frac{\mathrm{Vx}}{\sqrt{\operatorname{area}(M)}} ; \mathrm{Vy}^{\prime}=\frac{\mathrm{Vy}}{\sqrt{\text { area }(M)}} ; \mathrm{Vz}^{\prime}=\frac{\mathrm{Vz}}{\sqrt{\text { area }(M)}}$

where $\mathrm{Vx}, \mathrm{Vy}, \mathrm{Vz}$ are the coordinates of all vertices on the $\mathrm{X}$-axis, $\mathrm{Y}$-axis, and Z-axis, respectively; area $(M)$ is the surface area of object $M ; \mathrm{Vx}^{\prime}, \mathrm{Vy}^{\prime}, \mathrm{VZ}^{\prime}$ are the coordinates of transformed vertices on the $\mathrm{X}$-axis, $\mathrm{Y}$-axis, and Z-axis respectively.

\section{The shape asymmetry signature}

The LBO eigenvalues measure the intrinsic geometry of an object and are isometry invariant. Hence, the eigenvalue spectra are identical regardless of object positions, rotations, and mirroring (i.e., perfect projection from the brain structure of the right hemisphere to the left does not change the eigenvalue spectrum) $(28,49)$. Therefore, brain asymmetry can be calculated directly from the eigenvalue spectra of the two hemispheres (28) without image registration or smoothing $(28,49)$. In this study, after calculating the eigenvalues with surface area normalization, we subtracted the eigenvalue spectra of the right hemisphere from those of the left hemisphere in the same subject at each spatial scale (each eigenvalue index) to define the shape asymmetry signature (SAS). Formally, the SAS for subject $i$ is given by

$\Lambda^{i}=\lambda_{L}^{i}-\lambda_{R}^{i}$

where $\lambda$ is the eigenvalue spectrum $\lambda=\left(\lambda^{1}, \lambda^{2}, \ldots, \lambda^{n}\right)$ from the left $(L)$ and right $(R)$ hemispheres, each of which represents a certain spatial scale. There are other possible asymmetry indices $(10$, 77), but those indices are not appropriate for a surface area-normalized eigenvalue analysis (see SI Materials and Methods for details).

\section{Subject Identification}

Our first aim was to validate the SAS as a useful and robust measure of fluctuating asymmetry. We, therefore, evaluated the extent to which the SAS of each individual measure at time 1 (t1) could be used to identify the same person at time 2 (t2) in the longitudinal data, akin to a neuromorphological fingerprint. To this end, we revised the subject identification approach employed by Amico and Goni (58) and Mansour et al. (59). First, we computed the Pearson correlation between SAS vectors obtained for the $\mathrm{t} 1$ and $\mathrm{t} 2 \mathrm{scans}$ from the same subjects (diagonal elements of the Pearson correlation matrix in Fig. 1C), denoted by $r_{i i}$, as well as the correlation between $\mathrm{t} 1$ and $\mathrm{t} 2$ scans between different subjects, denoted by $\mathrm{r}_{i j}$ (off-diagonal elements of the Pearson correlation matrix in Fig. 1C).

To examine the within-subject and between-subject similarities (see Quantifying identifiability section in SI Materials and Methods for details), we utilized the Glass's $\Delta$, which is the standardized difference between the mean values of two categories of observations, normalized by the standard deviation of a control group (78), which is the between-subject group 
in our case. The Glass's $\Delta$ is recommended when the standard deviations of the two groups are substantially different $(78,79)$. Thus, our identification score was given by

Identification score $=\frac{\operatorname{mean}\left(r_{i i}\right)-\text { mean }\left(r_{i j}\right)}{S D\left(r_{i i}\right)}$

where $S D$ is the standard deviation. Higher scores indicate a greater capacity to discriminate between individuals. This approach allows for a more quantitative and finer-grained comparison of the identifiability of different metrics compared to other approaches that just rely on binary identification accuracy $(53,58,59)$.

\section{Identifying spatial scales for optimum subject identification}

Given a surface of $\mathrm{N}$ vertices, spectral shape analysis yields up to $\mathrm{N}$ eigenvalues, raising the question of how many eigenvalues constitute a sufficient description of cortical shape. Is a full representation of the entire surface necessary for optimal subject identification, or can this be achieved using a more compact set of eigenvalues? If so, the specific number of eigenvalues required would define the relevant spatial scale of shape differences that characterize the fluctuating asymmetry at which individual differences are most prevalent.

To address this question, we decompose the cortical surface and use an increasing number of eigenvalues, from the first two eigenvalues $\left(\lambda^{1}, \lambda^{2}\right)$ to the first 1000 eigenvalues $\left(\lambda^{1}, \lambda^{2}\right.$, $\lambda^{3}, \ldots, \lambda^{1000}$ ), each time computing the SAS and evaluating subject identifiability. For example, we first quantified the shape of cortical surface using only $\lambda^{1}$ and $\lambda^{2}$, thus capturing the coarsest scales of cortical shape. We then quantified the surface using $\lambda^{1}$ through $\lambda^{3}$, then $\lambda^{1}$ through $\lambda^{4}$, and so on. If there is a specific spatial scale that is optimal for this subject identification, we expect to see a peak in the identifiability score as a function of the truncation number, $k$. This peak not only defines the spatial scale at which individual variability, and thus fluctuating asymmetry, is most strongly expressed, but it also identifies a meaningful point at which to define a compressed summary of fluctuating asymmetry using the eigenvalue spectrum.

We evaluated the statistical significance of a given identifiability score at each value of $k$ using a permutation test in which we randomly shuffled the subject order in the t2 session 50,000 times. We compared the original identifiability score with the permuted peak score to calculate the $p$-value. Because of the $17 \mathrm{MZ}$ twin pairs in the HCP test-retest subsample, we kept the subjects' family structures intact $(55,56)$ when shuffling the HCP subjects. We also evaluated the identifiability performance of the SAS with respect to unihemispheric descriptors of size and shape: namely the eigenvalues (native, volume-normalized, or surface area-normalized) from the same hemispheres between time 1 and time 2 follow-up; concatenating eigenvalues of both left and right hemispheres between time 1 and time 2; and identifying the shape of one hemisphere from the shape of the other hemisphere both at time 1 or both at time 2 .

\section{Cortical shape harmonics}

The cerebral cortex is topologically equivalent to a sphere. Solving the Helmholtz equation for a sphere yields groups of eigenfunctions with the same eigenvalues and spatial wavelength, progressing along orthogonal axes (60). These groups in the solutions to the idealized spherical case are known as the spherical harmonics. The zeroth group $(L=0)$ is comprised of the first eigenvalue; the first group $(L=1)$ is comprised of the $2^{\text {nd }}, 3^{\text {rd }}$, and $4^{\text {th }}$ eigenvalues; the second group $(L=2)$ is comprised of the $5^{\text {th }}$ to $9^{\text {th }}$ eigenvalues, and so on. That is, there are $2(L+1)-1$ eigenvalues in the $L^{\text {th }}$ group. Robinson et al. (60) showed that while the eigenvalues between the cortical surface and sphere are different, the spherical grouping provides a rough division of the convoluted cortical surface. This is a useful grouping approach to investigate eigenfunctions and eigenvalues as the constituents of each group have roughly the same spatial wavelength. By averaging over several eigenvalues with similar spatial scales, we can also increase the stability of the truncation number across datasets. For example, the peak SAS identifiability appeared at the 
first 126 and 122 eigenvalues for the OASIS-3 and ADNI data, respectively, and these eigenvalues are all within the $11^{\text {th }}$ eigen-group $(L=11)$.

To estimate the corresponding wavelength of each eigen-group, we used an approximation of the spatial wavelength in the spherical case:

$W=\frac{2 \pi R s}{\sqrt{L(L+1)}}$

where $R s$ is the equivalent sphere of the original object (for the fsaverage case, $R s$ is about 67 $\mathrm{mm}$ ) and $L$ is the index of the eigen-group. We used the population-based template (fsaverage) as an example to show the wavelengths of the first 13 eigen-groups in Table S1.

\section{Non-shape descriptors of brain anatomical asymmetry and function}

To compare identifiability scores obtained with SAS compared to asymmetries for other morphological measurements, including volume, cortical thickness, and surface area, estimated using a method based on the commonly used definition $(13,26,42)$ as:

$A I^{S, i}=\frac{\left(P_{L}^{S, i}-P_{R}^{S, i}\right)}{0.5\left(P_{L}^{S, i}+P_{R}^{S, i}\right)}$

where $A I^{S, i}$ is the asymmetry index for parcellation $S$ of subject $i . P_{L}^{S, i}$ is the value of the morphological measurement from parcellation $S$ from subject i's left hemisphere; and $P_{R}^{S, i}$ is from the right hemisphere. To ensure that the asymmetry values were purely from the asymmetry effect and were not affected by the mean value of all brain regions (including both hemispheres), we adjusted the asymmetry index for the mean of each morphological measurement. Specifically, we revised the traditional asymmetry index by subtracting the mean value of the measurement across all parcellations of each subject before calculating the asymmetry index defined in Eq. 7 . This revised asymmetry measure $R A I^{S, i}$ explicitly calculated as:

$$
R A I^{S, i}=\frac{\left(P_{L}^{S, i}-M^{i}\right)-\left(P_{R}^{S, i}-M^{i}\right)}{0.5\left[\left(P_{L}^{S, i}-M^{i}\right)+\left(P_{R}^{S, i}-M^{i}\right)\right]}
$$

where $M^{i}$ is the mean value of the measurement across all regions in parcellation of subject $i$. We note that this is an important point, as without this correction, the asymmetry measure is dependent on the mean value (see SI Materials and Methods).

We employed a multi-modal parcellation, HCP-MMP1 version 1.0 (61) on the OASIS-3 subjects. We excluded one subject whose cortical surfaces could not be segmented by the HCPMMP1 atlas. There are 180 regions in each hemisphere of the HCP-MMP1 atlas, including the hippocampus that was excluded in our analysis. We created one vector per asymmetry-index measurement per subject and then used these asymmetry indices in the subject identifiability analyses.

We also compared the subject identifiability using the SAS versus functional connectivity (FC) from the subjects in the HCP test-retest subsample $(n=44$; we excluded one subject without REST1 data in one session) based on the Schaefer (62) and HCP-MMP1 (61) atlas (see SI Materials and Methods for details about FC estimation).

\section{Relationships with sex and handedness.}

Sex and handedness are two common characteristics that have been widely examined in the asymmetry literature $(3,4,7,10,11,13,14,27,28,32-36,63,80)$. We used a GLM to analyze relationships between each eigenvalue with sex and handedness on $1106 \mathrm{HCP}$ subjects (see SI 
Materials and Methods). Inference was performed via permutation testing with 100,000 iterations, as implemented in the Permutation Analysis of Linear Models (PALM) software package $(64,65)$, while accounting for the family structure of the data. We randomly shuffled the subjects' sex and handedness data, and the GLM was re-estimated at each iteration. False discovery rate correction $(q=0.05)$ was applied to correct for multiple comparisons.

\section{Heritability of brain shape}

The heritability of brain shape and cortical volume was obtained using a classical ACTE model applied to data from monozygotic (MZ) and same-sex dizygotic (DZ) twin pairs, and their non-twin siblings, from the HCP dataset (See SI Materials and Methods for details).

\section{Acknowledgments}

A.F. was supported by the Sylvia and Charles Viertel Foundation, National Health and Medical Research Council (IDs: 1197431 and 1146292), and Australian Research Council (ID: DP200103509). Data were provided in part by OASIS-3: Principal Investigators: T. Benzinger, D. Marcus, J. Morris; NIH P50 AG00561, P30 NS09857781, P01 AG026276, P01 AG003991, R01 AG043434, UL1 TR000448, R01 EB009352. AV-45 doses were provided by Avid Radiopharmaceuticals, a wholly owned subsidiary of Eli Lilly. Data were provided in part by the Human Connectome Project, WU-Minn Consortium (Principal Investigators: David Van Essen and Kamil Ugurbil; 1U54MH091657) funded by the $16 \mathrm{NIH}$ Institutes and Centers that support the NIH Blueprint for Neuroscience Research; and by the McDonnell Center for Systems Neuroscience at Washington University. Data collection and sharing for this project was funded by the Alzheimer's Disease Neuroimaging Initiative (ADNI) (National Institutes of Health Grant U01 AG024904) and DOD ADNI (Department of Defense award number W81XWH-12-2-0012). ADNI is funded by the National Institute on Aging, the National Institute of Biomedical Imaging and Bioengineering, and through generous contributions from the following: AbbVie, Alzheimer's Association; Alzheimer's Drug Discovery Foundation; Araclon Biotech; BioClinica, Inc.; Biogen; Bristol-Myers Squibb Company; CereSpir, Inc.; Cogstate; Eisai Inc.; Elan Pharmaceuticals, Inc.; Eli Lilly and Company; Eurolmmun; F. Hoffmann-La Roche Ltd and its affiliated company Genentech, Inc.; Fujirebio; GE Healthcare; IXICO Ltd.; Janssen Alzheimer Immunotherapy Research \& Development, LLC.; Johnson \& Johnson Pharmaceutical Research \& Development LLC.; Lumosity; Lundbeck; Merck \& Co., Inc.; Meso Scale Diagnostics, LLC.; NeuroRx Research; Neurotrack Technologies; Novartis Pharmaceuticals Corporation; Pfizer Inc.; Piramal Imaging; Servier; Takeda Pharmaceutical Company; and Transition Therapeutics. The Canadian Institutes of Health Research is providing funds to support ADNI clinical sites in Canada. Private sector contributions are facilitated by the Foundation for the National Institutes of Health (www.fnih.org). The grantee organization is the Northern California Institute for Research and Education, and the study is coordinated by the Alzheimer's Therapeutic Research Institute at the University of Southern California. ADNI data are disseminated by the Laboratory for Neuro Imaging at the University of Southern California. 


\section{References}

1. V. Duboc, P. Dufourcq, P. Blader, M. Roussigne, Asymmetry of the Brain: Development and Implications. Annu Rev Genet 49, 647-672 (2015).

2. O. Gunturkun, F. Strockens, S. Ocklenburg, Brain Lateralization: A Comparative Perspective. Physiol Rev 100, 1019-1063 (2020).

3. M. C. Corballis, I. S. Haberling, The Many Sides of Hemispheric Asymmetry: A Selective Review and Outlook. J Int Neuropsychol Soc 23, 710-718 (2017).

4. O. Gunturkun, S. Ocklenburg, Ontogenesis of Lateralization. Neuron 94, 249-263 (2017).

5. M. Esteves, E. Ganz, N. Sousa, H. Leite-Almeida, Asymmetrical Brain Plasticity: Physiology and Pathology. Neuroscience 10.1016/j.neuroscience.2020.01.022 (2020).

6. X. Li, T. J. Crow, W. D. Hopkins, Q. Gong, N. Roberts, Human torque is not present in chimpanzee brain. Neuroimage 165, 285-293 (2018).

7. A. W. Toga, P. M. Thompson, Mapping brain asymmetry. Nat Rev Neurosci 4, 37-48 (2003).

8. L. Zhao, W. Matloff, Y. Shi, R. P. Cabeen, A. W. Toga, Mapping Complex Brain Torque Components and Their Genetic and Phenomic Architecture in 24,112 healthy individuals. bioRxiv [Preprint] (2021). https://doi.org/10.1101/2021.03.09.434625 (Accessed 13 September 2021).

9. X. Kang, T. J. Herron, M. Ettlinger, D. L. Woods, Hemispheric asymmetries in cortical and subcortical anatomy. Laterality 20, 658-684 (2015).

10. X. Z. Kong et al., Mapping cortical brain asymmetry in 17,141 healthy individuals worldwide via the ENIGMA Consortium. Proc Natl Acad Sci U S A 115, E5154-E5163 (2018).

11. K. J. Plessen, K. Hugdahl, R. Bansal, X. Hao, B. S. Peterson, Sex, age, and cognitive correlates of asymmetries in thickness of the cortical mantle across the life span. $J$ Neurosci 34, 6294-6302 (2014).

12. A. Gomez-Robles, W. D. Hopkins, C. C. Sherwood, Increased morphological asymmetry, evolvability and plasticity in human brain evolution. Proc Biol Sci 280, 115-126 (2013).

13. X. Z. Kong et al., Mapping brain asymmetry in health and disease through the ENIGMA consortium. Hum Brain Mapp 10.1002/hbm.25033 (2020).

14. A. Deep-Soboslay et al., Handedness, heritability, neurocognition and brain asymmetry in schizophrenia. Brain 133, 3113-3122 (2010).

15. M. C. Postema et al., Altered structural brain asymmetry in autism spectrum disorder in a study of 54 datasets. Nat Commun 10, 4958 (2019).

16. A. Gomez-Robles, W. D. Hopkins, S. J. Schapiro, C. C. Sherwood, The heritability of chimpanzee and human brain asymmetry. Proc Biol Sci 283 (2016).

17. Y. Cai et al., Grey matter volume abnormalities in patients with bipolar I depressive disorder and unipolar depressive disorder: a voxel-based morphometry study. Neurosci Bull 31, 4-12 (2015).

18. A. Ferro et al., Longitudinal investigation of the parietal lobe anatomy in bipolar disorder and its association with general functioning. Psychiatry Res Neuroimaging 267, 22-31 (2017).

19. B. W. Fling, G. G. Dutta, H. Schlueter, M. H. Cameron, F. B. Horak, Associations between Proprioceptive Neural Pathway Structural Connectivity and Balance in People with Multiple Sclerosis. Front Hum Neurosci 8, 814 (2014).

20. C. Nunez et al., Global brain asymmetry is increased in schizophrenia and related to avolition. Acta Psychiatr Scand 135, 448-459 (2017).

21. N. Okada et al., Abnormal asymmetries in subcortical brain volume in schizophrenia. Mol Psychiatry 21, 1460-1466 (2016).

22. N. Okada et al., Abnormal asymmetries in subcortical brain volume in early adolescents with subclinical psychotic experiences. Transl Psychiatry 8, 254 (2018). 
23. C. Wachinger, D. H. Salat, M. Weiner, M. Reuter, I. Alzheimer's Disease Neuroimaging, Whole-brain analysis reveals increased neuroanatomical asymmetries in dementia for hippocampus and amygdala. Brain 139, 3253-3266 (2016).

24. M. Ribolsi, Z. J. Daskalakis, A. Siracusano, G. Koch, Abnormal asymmetry of brain connectivity in schizophrenia. Front Hum Neurosci 8, 1010 (2014).

25. Z. Peng et al., Cortical asymmetries in unaffected siblings of patients with obsessivecompulsive disorder. Psychiatry Res 234, 346-351 (2015).

26. F. Kurth, P. M. Thompson, E. Luders, Investigating the differential contributions of sex and brain size to gray matter asymmetry. Cortex 99, 235-242 (2018).

27. C. Nunez et al., A large-scale study on the effects of sex on gray matter asymmetry. Brain Struct Funct 223, 183-193 (2018).

28. C. Wachinger et al., BrainPrint: a discriminative characterization of brain morphology. Neuroimage 109, 232-248 (2015).

29. V. A. Kovalev, F. Kruggel, D. Y. von Cramon, Gender and age effects in structural brain asymmetry as measured by MRI texture analysis. Neurolmage 19, 895-905 (2003).

30. T. Guadalupe et al., Asymmetry within and around the human planum temporale is sexually dimorphic and influenced by genes involved in steroid hormone receptor activity. Cortex 62, 41-55 (2015).

31. H. Takao et al., Gray and white matter asymmetries in healthy individuals aged 21-29 years: a voxel-based morphometry and diffusion tensor imaging study. Hum Brain Mapp 32, 1762-1773 (2011).

32. K. L. Narr et al., Asymmetries of cortical shape: Effects of handedness, sex and schizophrenia. Neuroimage 34, 939-948 (2007).

33. H. Steinmetz, J. Volkmann, L. Jancke, H. J. Freund, Anatomical Left-Rght Asymmetry of Languagerelated Temporal Cortex Is Different in Left- and Right-handers. Annals of Neurology 29, 315-319 (1991).

34. C. D. Good et al., Cerebral asymmetry and the effects of sex and handedness on brain structure: a voxel-based morphometric analysis of 465 normal adult human brains. Neuroimage 14, 685-700 (2001).

35. T. Guadalupe et al., Differences in cerebral cortical anatomy of left- and right-handers. Front Psychol 5, 261 (2014).

36. S. Maingault, N. Tzourio-Mazoyer, B. Mazoyer, F. Crivello, Regional correlations between cortical thickness and surface area asymmetries: A surface-based morphometry study of 250 adults. Neuropsychologia 93, 350-364 (2016).

37. S. Neubauer, P. Gunz, N. A. Scott, J. J. Hublin, P. Mitteroecker, Evolution of brain lateralization: A shared hominid pattern of endocranial asymmetry is much more variable in humans than in great apes. Sci Adv 6, eaax9935 (2020).

38. C. C. Sherwood, A. Gómez-Robles, Brain Plasticity and Human Evolution. Annual Review of Anthropology 46, 399-419 (2017).

39. C. Francks, Exploring human brain lateralization with molecular genetics and genomics. Ann N Y Acad Sci 1359, 1-13 (2015).

40. C. G. F. de Kovel, S. N. Lisgo, S. E. Fisher, C. Francks, Subtle left-right asymmetry of gene expression profiles in embryonic and foetal human brains. Sci Rep 8, 12606 (2018).

41. J. Graham, B. Özener, Fluctuating Asymmetry of Human Populations: A Review. Symmetry 8 (2016).

42. Z. Sha et al., The genetic architecture of structural left-right asymmetry of the human brain. Nat Hum Behav 10.1038/s41562-021-01069-w (2021).

43. A. Gomez-Robles, L. D. Reyes, C. C. Sherwood, Landmarking Brains. 10.1007/978-4431-56582-6_8, 115-126 ( 2018).

44. L. Xiang, T. J. Crow, W. D. Hopkins, N. Roberts, Comparison of Surface Area and Cortical Thickness Asymmetry in the Human and Chimpanzee Brain. Cereb Cortex 10.1093/cercor/bhaa202 (2020).

45. F. Kruggel, A. Solodkin, Heritability of Structural Patterning in the Human Cerebral Cortex. Neuroimage 221, 117169 (2020). 
46. F. Kurth, C. Gaser, E. Luders, A 12-step user guide for analyzing voxel-wise gray matter asymmetries in statistical parametric mapping (SPM). Nat Protoc 10, 293-304 (2015).

47. M. Reuter, F. E. Wolter, M. Shenton, M. Niethammer, Laplace-Beltrami Eigenvalues and Topological Features of Eigenfunctions for Statistical Shape Analysis. Comput Aided Des 41, 739-755 (2009).

48. T. Ge et al., Multidimensional heritability analysis of neuroanatomical shape. Nat Commun 7, 13291 (2016).

49. M. Reuter, F.-E. Wolter, N. Peinecke, Laplace-Beltrami spectra as 'Shape-DNA' of surfaces and solids. Computer-Aided Design 38, 342-366 (2006).

50. B. Lévy, Laplace-beltrami eigenfunctions towards an algorithm that "understands" geometry. IEEE International Conference on Shape Modeling and Applications - SMI 2006 10.1109/SMI.2006.21, 13 (2006).

51. R. Richards et al., Increased hippocampal shape asymmetry and volumetric ventricular asymmetry in autism spectrum disorder. Neuroimage Clin 26, 102207 (2020).

52. C. Wachinger et al., A Longitudinal Imaging Genetics Study of Neuroanatomical Asymmetry in Alzheimer's Disease. Biol Psychiatry 84, 522-530 (2018).

53. E. S. Finn et al., Functional connectome fingerprinting: identifying individuals using patterns of brain connectivity. Nat Neurosci 18, 1664-1671 (2015).

54. P. J. LaMontagne et al., OASIS-3: Longitudinal Neuroimaging, Clinical, and Cognitive Dataset for Normal Aging and Alzheimer Disease. medRxiv [Preprint] (2019). https://doi.org/10.1101/2019.12.13.19014902 (Accessed 13 September 2021).

55. D. C. Van Essen et al., The WU-Minn Human Connectome Project: an overview. Neuroimage 80, 62-79 (2013).

56. B. Fischl et al., Whole Brain Segmentation Automated Labeling of Neuroanatomical Structures in the Human Brain. Neuron 33, 341-355 (2002).

57. M. F. Glasser et al., The minimal preprocessing pipelines for the Human Connectome Project. Neuroimage 80, 105-124 (2013).

58. E. Amico, J. Goni, The quest for identifiability in human functional connectomes. Sci Rep 8, 8254 (2018).

59. L. S. Mansour, Y. Tian, B. T. T. Yeo, V. Cropley, A. Zalesky, High-resolution connectomic fingerprints: Mapping neural identity and behavior. Neuroimage 229, 117695 (2021).

60. P. A. Robinson et al., Eigenmodes of brain activity: Neural field theory predictions and comparison with experiment. Neuroimage 142, 79-98 (2016).

61. M. F. Glasser et al., A multi-modal parcellation of human cerebral cortex. Nature 536, 171-178 (2016).

62. A. Schaefer et al., Local-Global Parcellation of the Human Cerebral Cortex from Intrinsic Functional Connectivity MRI. Cereb Cortex 28, 3095-3114 (2018).

63. X. Z. Kong et al., Large-Scale Phenomic and Genomic Analysis of Brain Asymmetrical Skew. Cereb Cortex 10.1093/cercor/bhab075 (2021).

64. A. M. Winkler et al., Non-parametric combination and related permutation tests for neuroimaging. Hum Brain Mapp 37, 1486-1511 (2016).

65. A. M. Winkler, M. A. Webster, D. Vidaurre, T. E. Nichols, S. M. Smith, Multi-level block permutation. Neuroimage 123, 253-268 (2015).

66. C. H. Chen et al., Genetic influences on cortical regionalization in the human brain. Neuron 72, 537-544 (2011).

67. C. H. Chen et al., Hierarchical genetic organization of human cortical surface area. Science 335, 1634-1636 (2012).

68. C. H. Chen et al., Genetic topography of brain morphology. Proc Natl Acad Sci U S A 110, 17089-17094 (2013).

69. L. T. Eyler et al., Conceptual and data-based investigation of genetic influences and brain asymmetry: a twin study of multiple structural phenotypes. J Cogn Neurosci 26, 11001117 (2014).

70. A. Felton et al., Bilingualism Influences Structural Indices of Interhemispheric Organization. J Neurolinguistics 42, 1-11 (2017). 
71. L. Ronan, P. C. Fletcher, From genes to folds: a review of cortical gyrification theory. Brain Struct Funct 220, 2475-2483 (2015).

72. F. Kruggel, The macro-structural variability of the human neocortex. Neuroimage 172, 620-630 (2018).

73. S. L. Valk et al., Shaping brain structure Genetic and phylogenetic axes of macroscale organization of cortical thickness. Science Advances 6, eabb3417 (2020).

74. Z. Lian et al., A comparison of methods for non-rigid 3D shape retrieval. Pattern Recognition 46, 449-461 (2013).

75. S. Boker et al., OpenMx: An Open Source Extended Structural Equation Modeling Framework. Psychometrika 76, 306-317 (2011).

76. M. C. Neale et al., OpenMx 2.0: Extended Structural Equation and Statistical Modeling. Psychometrika 81, 535-549 (2016).

77. J. E. Moodie et al., Fluctuating asymmetry in brain structure and general intelligence in 73-year-olds. Intelligence 78, 101407 (2020).

78. G. V. Glass, B. McGaw, M. L. Smith, Meta-Analysis in Social Research. (1981).

79. D. Lakens, Calculating and reporting effect sizes to facilitate cumulative science: a practical primer for t-tests and ANOVAs. Front Psychol 4, 863 (2013).

80. I. Savic, Asymmetry of cerebral gray and white matter and structural volumes in relation to sex hormones and chromosomes. Front Neurosci 8, 329 (2014).

81. A. Arnatkeviciute et al., Genetic influences on hub connectivity of the human connectome. Nat Commun 12, 4237 (2021).

82. M. Allen, D. Poggiali, K. Whitaker, T. R. Marshall, R. A. Kievit, Raincloud plots: a multiplatform tool for robust data visualization. Wellcome Open Res 4, 63 (2019).

\section{Figures and Tables}


bioRxiv preprint doi: https://doi.org/10.1101/2021.09.14.460242; this version posted September 15, 2021. The copyright holder for this preprint (which was not certified by peer review) is the author/funder, who has granted bioRxiv a license to display the preprint in perpetuity. It is made available under aCC-BY-NC 4.0 International license.
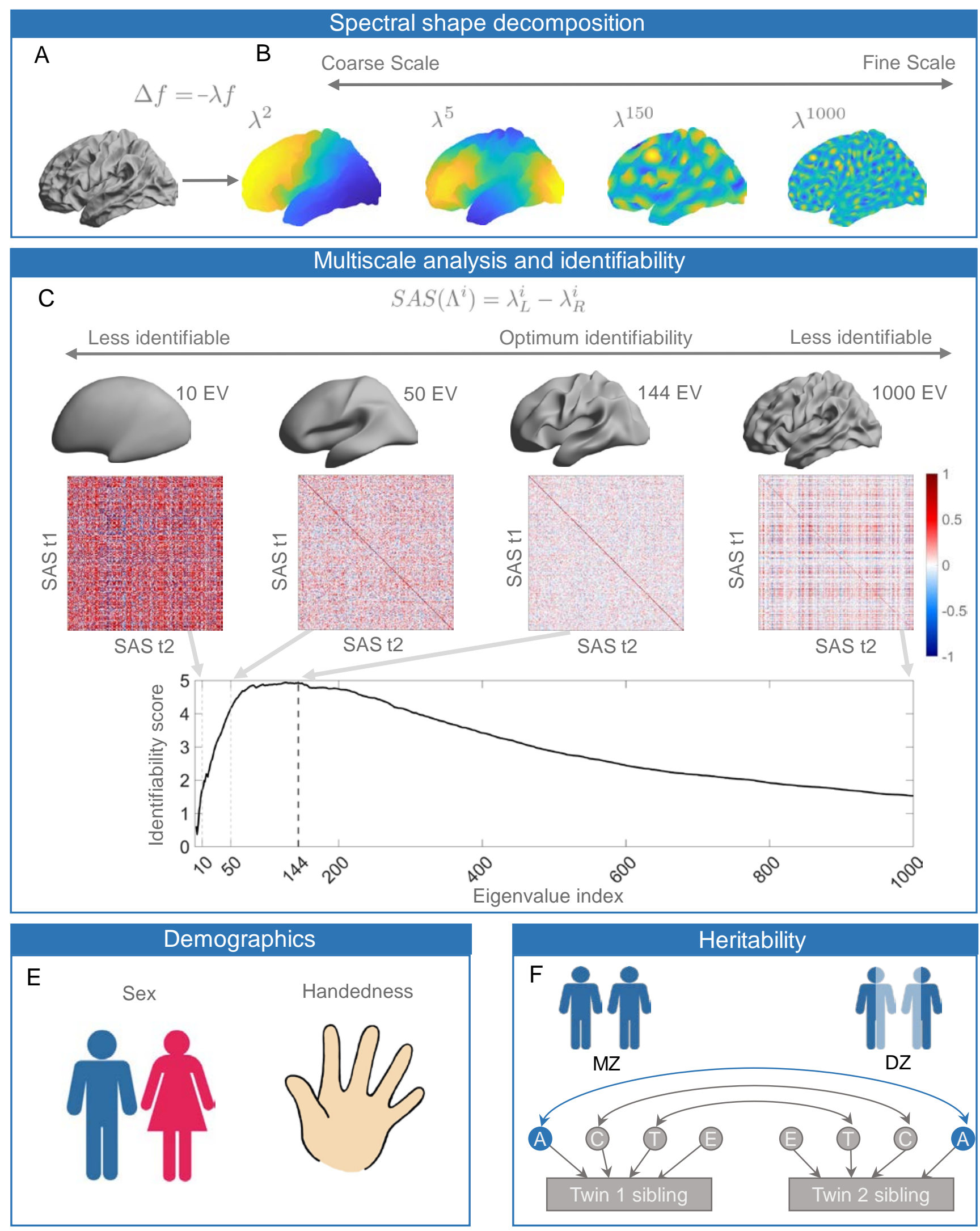
Figure 1. Schematic of our analysis workflow. $(A)$ The shapes of the left and right hemispheres are independently analyzed using the Laplace-Beltrami Operator (LBO) via the Shape-DNA algorithm $(47,49)$. (B) Eigenfunctions of the LBO are obtained by solving the Helmholtz equation on the surface, given by $\Delta f=-\lambda f$, where $f$ is corresponds to a distinct eigenfunction, and $\lambda$ is the corresponding eigenvalue. Each eigenvalue $\lambda^{i}, i=1,2, \ldots, 1000$, quantifies the degree to which a given eigenfunction is expressed in the intrinsic geometry of the cortex. Higher-order eigenvalues describe shape variations at finer spatial scales. $(C)$ The Shape Asymmetry Signature (SAS) is quantified as the difference in the left and right hemisphere eigenspectra, providing a summary measure of multiscale cortical shape asymmetries. To investigate the identifiability of the SAS, we use Pearson's correlation to calculate the similarity between the SAS vectors obtained for the time 1 (t1) and time 2 (t2) 2 scans from the same individuals (diagonal elements of the matrices) as well as the correlation between $\mathrm{t} 1$ and $\mathrm{t} 2$ scans between different subjects (off-diagonal elements). We estimate identifiability by first correlating the initial two eigenvalues, then the initial three eigenvalues, and so on to a maximum of 1000 eigenvalues. Here we show example identifiability matrices obtained when using the first 10, 50, 144, and 1000 eigenvalues, and cortical surface reconstructions showing the resolution of shape variations captured by each scale. $(D)$ Repeating the identifiability analysis up to a maximum of 1000 eigenvalues yields a curve with a clear peak, representing the scale at which individual differences in cortical shape are maximal. For the SAS, this peak occurs when the first 144 eigenvalues are used (black dashed line), which offers a fairly coarse description of shape variations (see panel $C$ ). We then use a similar analysis approach to investigate associations between scale-specific shape variations and $(E)$ sex, handedness as well as $(F)$ heritability. The data in this figure are from the OASIS-3 $(n=233)$ cohort, and the cortical surfaces are from a population-based template (fsaverage in FreeSurfer). Panel $F$ was adapted from (81). 

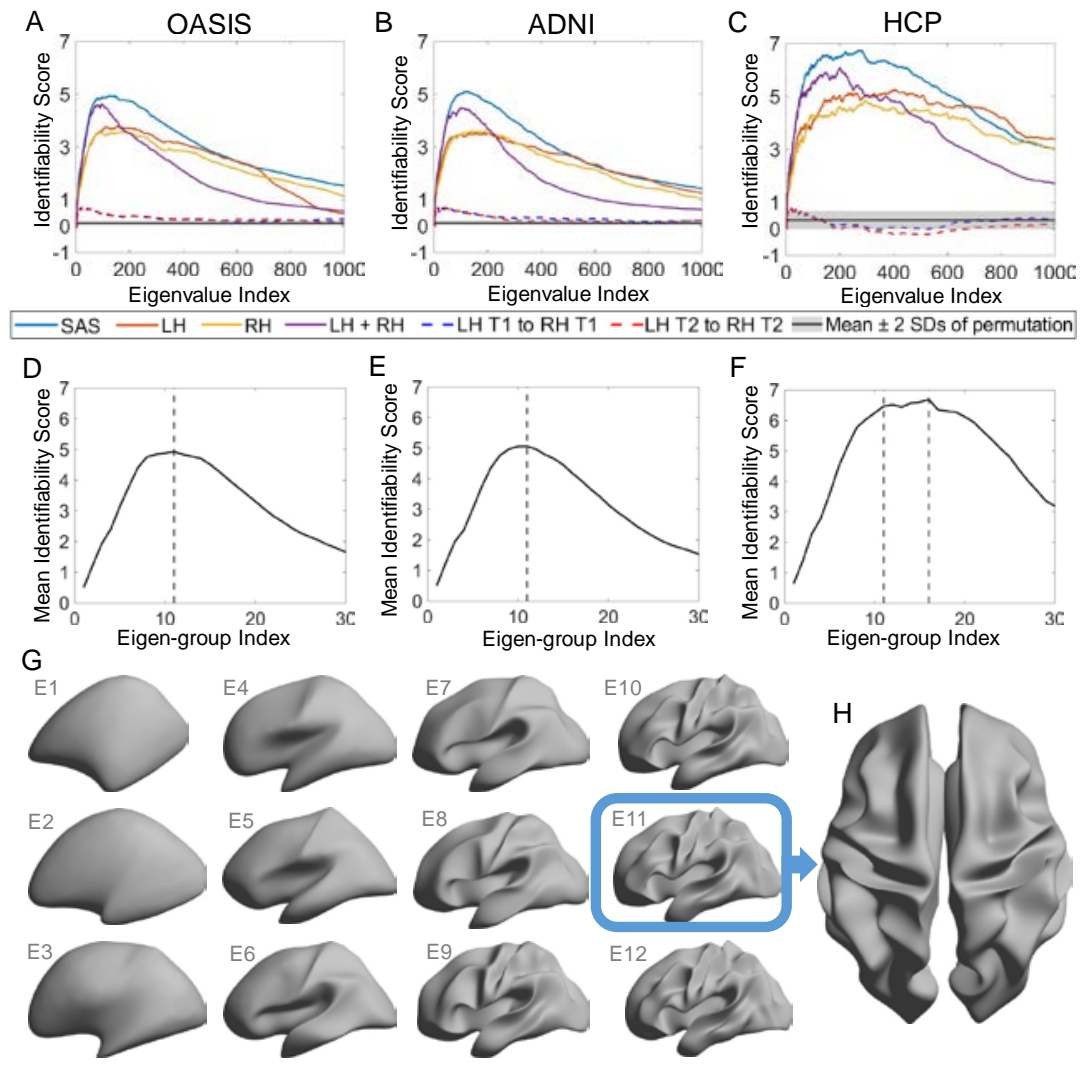

Figure 2. Identifiability of different shape descriptors at different spatial scales. $(A-C)$ Identifiability scores for shape features across eigenvalue indices. The identifiability scores of the shape asymmetry signature (SAS) are generally higher than the scores for shape descriptors of individual hemispheres or scores obtained when concatenating both hemispheres. The SAS scores are also much higher than the scores obtained by randomly shuffling the order of the subjects at time 2 (shaded area represents mean \pm 2 SDs). $(D-F)$ The cumulative mean identifiability scores for each eigenvalue group, derived from correspondence with spherical harmonics (60). The peak mean identifiability occurs at the $11^{\text {th }}$ eigenvalue group for the OASIS-3 $(D)$ and ADNI data $(E)$, representing the first 144 eigenvalues. The curve of the mean identifiability score for the HCP data $(F)$ flattens after the $11^{\text {th }}$ group and peaks at the $16^{\text {th }}$ group. $(G)$ Cortical surfaces reconstructed at different spatial scales, starting with only the first eigen-group (left) and incrementally adding more groups to a maximum of the first 12 eigen-groups. $(H)$ Overhead view of the spatial scale corresponding to the eigen-group at which identifiability is maximal in the OASIS-3 and ADNI datasets (i.e., the first 11 eigen-groups, corresponding to the first 144 eigenvalues). 


\section{Morphology}

A

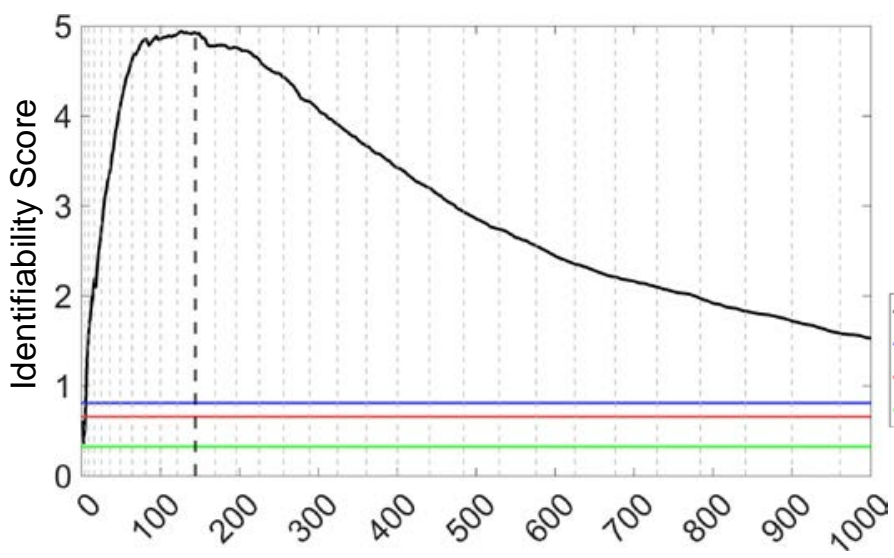

Eigenvalue Index

\section{Function}

C

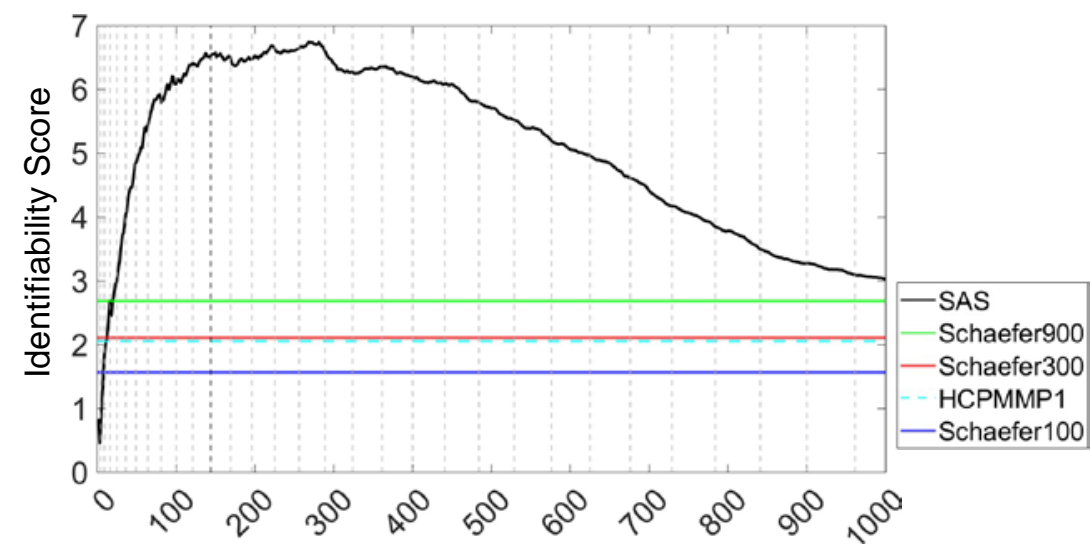

Eigenvalue Index
B

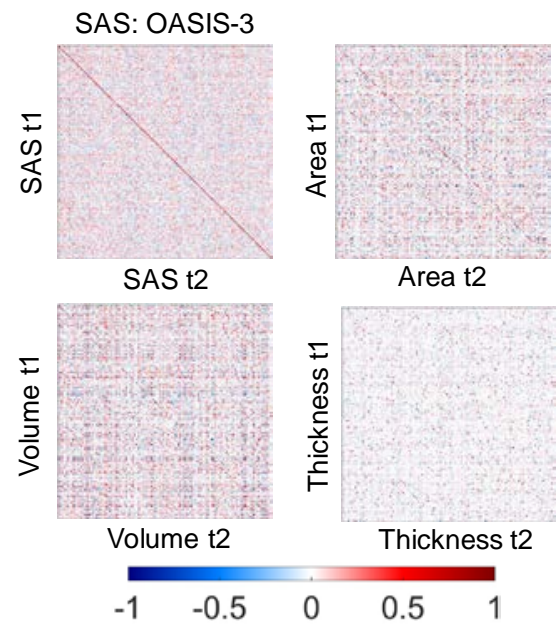

D

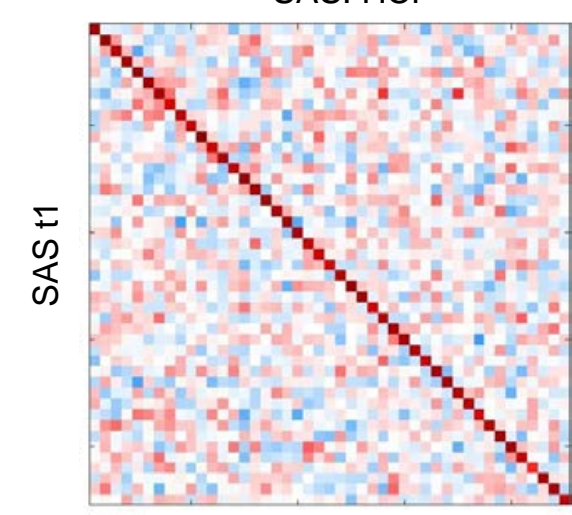

SAS t2

E Coarse resolution

Fine resolution

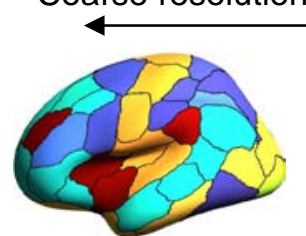

F

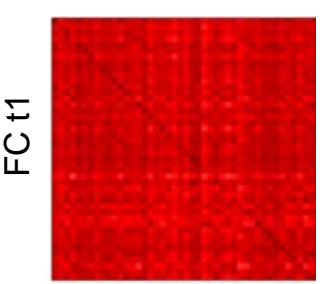

FC t2

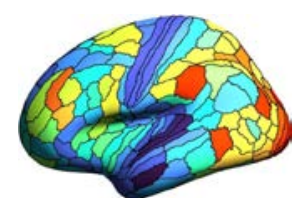

HCP-MMP1

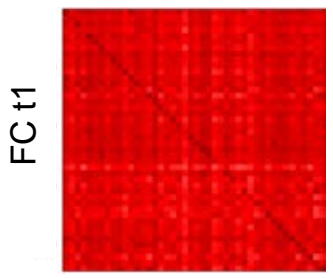

FC t2

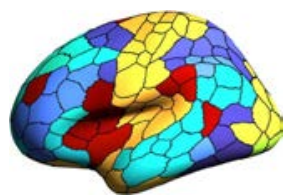

Schaefer 300

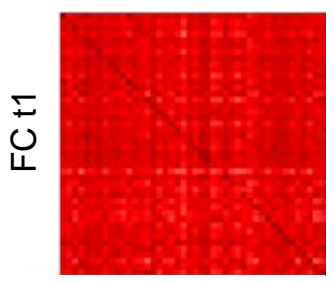

FC t2

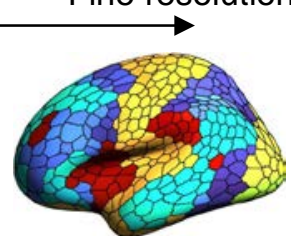

Schaefer 900

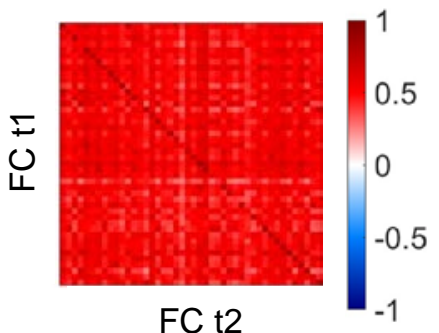


bioRxiv preprint doi: https://doi.org/10.1101/2021.09.14.460242; this version posted September 15, 2021. The copyright holder for this preprint (which was not certified by peer review) is the author/funder, who has granted bioRxiv a license to display the preprint in perpetuity. It is made available under aCC-BY-NC 4.0 International license.

Figure 3. Cortical shape asymmetries are more identifiable than size-related descriptors or functional connectivity. $(A)$ Identifiability scores for the SAS are higher than those obtained for asymmetries based on cortical surface area (identifiability score $=0.81$ ), volume (identifiability score $=0.66)$, and thickness (identifiability score $=0.33)$ for the OASIS-3 dataset $(n=232 ;$ See $S I$ Materials and Methods). (B) Matrix of the Pearson correlation coefficients for shape asymmetry signatures and size-based morphological asymmetries from MRI scans taken at different time points (t1 and $\mathrm{t} 2$ ) of the OASIS-3 subjects. (C) SAS identifiability is higher than the identifiability based on functional connectivity, assessed with parcellations at different resolution scales in the HCP test-retest dataset $(n=44)$. $(D)$ Matrix of the Pearson correlation coefficients for shape asymmetry signatures of the HCP subjects. $(E)$ Four resolution scales of parcellations used in the functional connectivity analysis (shown on an inflated fsaverage surface in FreeSurfer). ( $F$ ) Matrix of the Pearson correlation coefficients for functional connectivity using the Schaefer 100 (identifiability score $=1.57$ ), HCP-MMP1 (identifiability score $=2.06$ ), Schaefer 300 (identifiability score $=2.11$ ), and Schaefer 900 (identifiability score $=2.69$ ) parcellations. 
bioRxiv preprint doi: https://doi.org/10.1101/2021.09.14.460242; this version posted September 15, 2021. The copyright holder for this preprint (which was not certified by peer review) is the author/funder, who has granted bioRxiv a license to display the preprint in perpetuity. It is made available under aCC-BY-NC 4.0 International license.

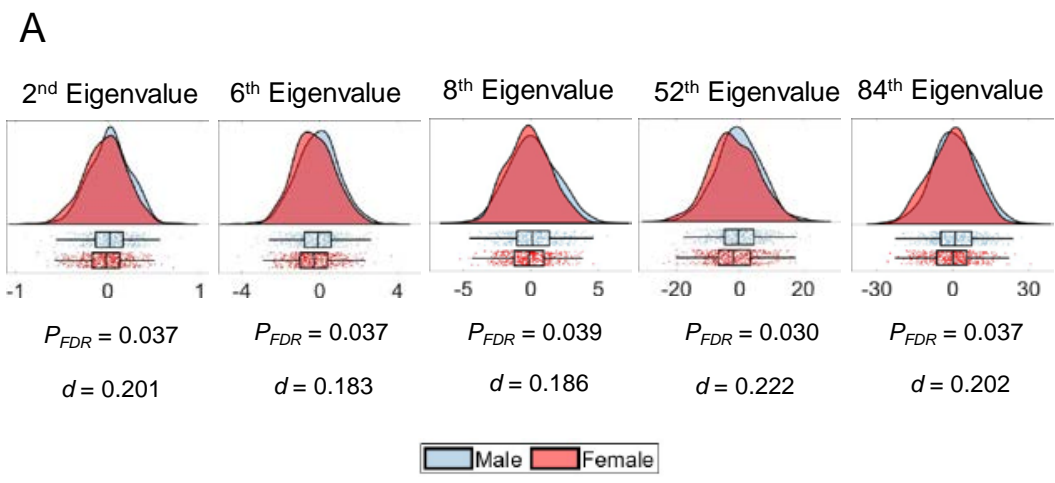

B

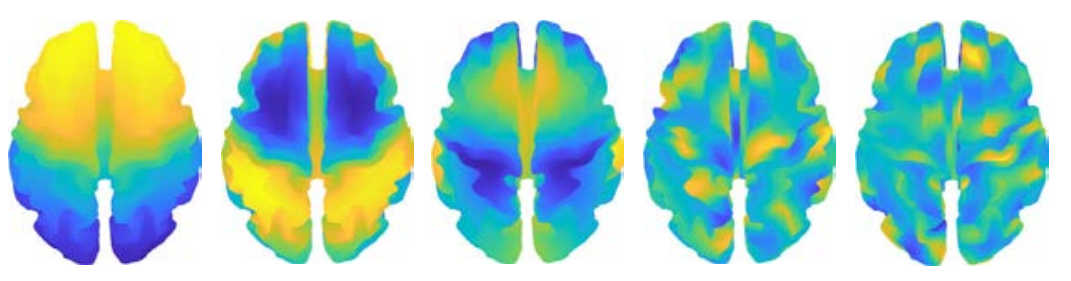

Figure 4. Sex differences in eigenvalue asymmetries. $(A)$ Smoothed distributions and boxplots with mean and interquartile range (82) of the eigenvalues among males $(n=504)$ and females $(n=$ 602). Under these five spatial scales, female brains show a greater rightward asymmetry than males. The $p$-values are FDR-corrected values of the correlation between sex and SAS, obtained via a GLM. The $d$ values are effect sizes (Cohen's $d$ ). (B) The corresponding eigenfunction of each eigenvalue in panel $A$ shown on a population-based template. 
bioRxiv preprint doi: https://doi.org/10.1101/2021.09.14.460242; this version posted September 15, 2021. The copyright holder for this preprint (which was not certified by peer review) is the author/funder, who has granted bioRxiv a license to display the preprint in perpetuity. It is made available under aCC-BY-NC 4.0 International license.
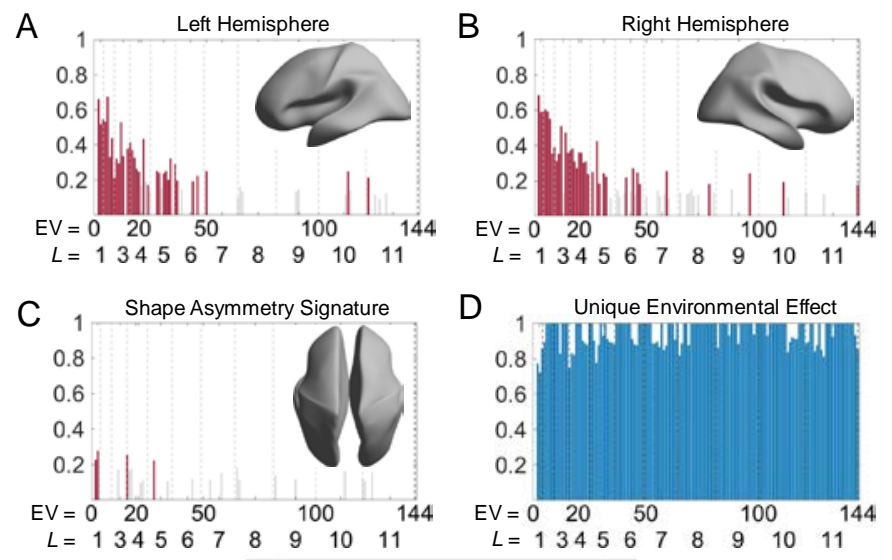

Figure 5. Heritability of cortical shape. $(A-B)$ Heritability of the eigenvalues of the left $(A)$ and right $(B)$ hemispheres. The insets show the corresponding spatial scales by reconstructing the surfaces using the first six eigen-groups. (C) Heritability of the SAS. The inset shows the corresponding spatial scale with some level of genetic influence, obtained by reconstructing the surface using the first five eigen-groups. $(D)$ Unique environmental influences to the SAS at each eigenvalue. Statistical significance is evaluated after FDR-correction. Note that significance is not estimated for unique environmental effects as this represents the reference model against which other genetically informed models are compared. We use 79 same-sex DZ twin pairs, $138 \mathrm{MZ}$ twin pairs, and 160 of their non-twin siblings. 